Methods 1082 patients meeting entry criteria for OA, responsive to NSAIDs, were randomised 3:3:1 to treatment with rofecoxib $25 \mathrm{mg}$ qd $(\mathrm{n}=471)$, celecoxib $200 \mathrm{mg}$ qd $(\mathrm{n}=460)$ or placebo $(\mathrm{n}=151)$. Efficacy was assessed over the first 6 days of therapy and at weeks 2, 4, and 6 by WOMAC questionnaire and patient global assessment of response to therapy (PGART).

Results Demographics were well balanced. Significantly more patients on placebo discontinued prematurely compared to both active groups ( $p<0.001)$ mainly due to lack of efficacy. Rofecoxib provided statistically superior relief of night pain $(\mathrm{p}=$ $0.023)$, morning stiffness $(\mathrm{p}=0.002)$, rest pain $(\mathrm{p}=0.023)$, and walking pain $(\mathrm{p}=0.005)$ compared to celecoxib. Rofecoxib was significantly superior to celecoxib on all WOMAC subsacles, including pain $(\mathrm{p}=0.008)$, stiffness $(\mathrm{p}=0.001)$ and physical function $(\mathrm{p}=0.01)$. Rofecoxib was superior to celecoxib in $\%$ of patients with good or excellent PGART over 6 weeks $(\mathrm{p}=$ 0.014 ) and provided quicker onset of efficacy as assessed by time to first report of good or excellent response $(p<0.001)$. Both active groups were superior to placebo on efficacy endpoints. Incidence of clinical AEs, drug related AEs, serious AEs, and discontinuations due to AEs was similar between active groups.

Conclusion In this study, once daily doses of rofecoxib provided superior relief of pain and other related symptoms in OA compared to celecoxib and placebo. All treatments were generally well tolerated.

\section{SAT0090 CORRELATION BETWEEN PROXIMAL FEMUR BONE MINERAL DENSITY AND HIP OSTEOARTHRITIS: PRELIMINARY DATA}

N Malavolta, P Mastrapasqua, L Lisi, M Frigato, MG Prandin, S Gnudi. Medicina Interna E dell'Invecchiamento, Azienda Ospedaliera Di Bologna-Policlinico S. Orsola -Malpighi, Bologna, Italy

\subsection{6/annrheumdis-2001.465}

Background Osteoarthritis (OA) is a very frequent disease in postmenopausal women. The question whether high bone mineral density (BMD) plays a role in the development of this disease is under investigation.

Objectives Here we report the preliminary data of a study aimed at investigating the relationship between the BMD of healthy women and women with OA of the hip.

Methods Exclusion criteria included all diseases and drugs known to cause BMD loss. Till now 136 age matched women have entered the study: 46 with OA (mean age 65.76 , SD 8.07) and 92 healthy individuals (mean age 65.60, SD 8.12). All subjects underwent Dual Energy X-ray Absorptiometry (DEXA-XR 36 Norland) measurements of the femoral neck, trochanter, Ward?s and the lumbar spine (L2-L4). Age, age of menopause, height and weight were also collected. Data were analysed using the Anova test to compare groups. The ability of selected parameters to discriminate the two groups of patients was tested by logistic regression.

Results Weight $(p=0.003)$ and femoral neck BMD ( $p=0.045)$ were significantly higher in women with OA than in healthy women. None of the other variables were significantly different in the two groups. At the logistic regression both age corrected weight (OR: 1.05 ; 95\% CI: 1.01-1.09) and age corrected femoral neck BMD (OR: 1.01; 95\% CI: 1.00-1.03) were able to significantly discriminate the two groups of women. Both variables correctly classified $13.04 \%$ of OA while $93.48 \%$ of healthy women were correctly classified by the variable weight and 97.83\% the femoral neck BMD.

Conclusion Our data suggest that the body weight and femoral neck BMD are the main risk factors for OA of the hip. The fact that at the trochanter and at the Ward?s (where BMD measurement does not discriminate between the two groups) the cortical component of bone is lower than at the femoral neck should indicate that high cortical bone density is a marker of OA.

\section{SAT0091 EFFECTS OF JOINT LAVAGE AND HYALURONIC ACID INFILTRATION IN PATIENTS WITH OSTEOARTHRITIS OF THE KNEE}

MA Caracuel, MC Muñoz-Villanueva, A Escudero, R Veroz, G Frias, J Vacas, C Perez, M Romero, V Perez, J Gonzalez, FG Martinez, E Collantes. Rheumatology Service, Hospital Universitario Reina Sofia, Cordoba, Spain

\subsection{6/annrheumdis-2001.466}

Background Infiltration with hyaluronic acid (HA) and joint lavage (JL) have recently been proposed as local treatments for osteoarthritis of the knee. Joint lavage is a therapeutic treatment involving passing cold sterile $0.9 \%$ saline through the knee joint in order to have the liquid reach the inside of the joint capsule. Objectives To evaluate the efficacy of joint lavage and intraarticular infiltration with hyaluronic acid, alone and in combination (JLHA), as symptomatic treatments for knee osteoarthritis.

Methods An overall 37 patients (8 males and 29 females) aged 43-85 years with knee osteoarthritis of radiological grade II on the Kellgren scale were randomly classified into three thera-peutic groups, namely: JL $(\mathrm{n}=14)$, HA $(\mathrm{n}=10)$ and JLHA $(\mathrm{n}=$ 13). The patients in group $\mathrm{JL}$ received joint lavage, on day 0 only; those in group JLHA were subjected to joint lavage on day 0 and to infiltration on days $0,7,14,21$ and 28. Outcome measures evaluated at baseline, at days 7 and 30 included severity of pain (VAS), Global status (VAS) and Lequesne?s functional index.

Results The results of the Global Status difference from baseline are summarised in the following Table 1:

\begin{tabular}{lll} 
Abstract & SAT0091 Table 1 & \\
\hline & $\begin{array}{l}\text { Assessment at week } \\
\text { Differential Global Status } \\
\text { (significance level) }\end{array}$ & $\begin{array}{l}\text { Assessment at month } \\
\text { Differential Global Status } \\
\text { (significance level) }\end{array}$ \\
\hline JL vs HA & -5.14 vs $-3.10(p=0.03)$ & -5.07 vs $-2.80(p=0.02)$ \\
HA vs & -3.10 vs $-5.38(p=0.01)$ & -2.80 vs $-5.76(p=0.02)$ \\
JLHA & & -5.07 vs $-5.76(p=0.36)$ \\
JL vs & -5.14 vs $-5.38(p=0.59)$ & \\
JLHA & &
\end{tabular}

Conclusion Our results suggest significant differences between the tested treatments, Joint lavage alone and followed by infiltration with the hyaluronic acid is more effective than local infiltration with hyaluronic acid alone. 